\title{
Multilinguales
}

15 | 2021

Recherches algériennes en sociolinguistique et en littérature : discours scientifique/discours militant, quelles frontières?

\section{Une lecture de la reception du roman de langue kabyle (ou l'ungal)}

A lecture on the reception of the berber (kabyle) novel

\section{Nabila Sadi}

\section{(2) OpenEdition}

Journals

Édition électronique

URL : https://journals.openedition.org/multilinguales/6064

DOI : $10.4000 /$ multilinguales.6064

ISSN : 2335-1853

Éditeur

Université Abderrahmane Mira - Bejaia

\section{Référence électronique}

Nabila Sadi, « Une lecture de la reception du roman de langue kabyle (ou l'ungal) », Multilinguales [En ligne], 15 | 2021, mis en ligne le 15 juin 2021, consulté le 14 février 2022. URL : http://

journals.openedition.org/multilinguales/6064; DOI : https://doi.org/10.4000/multilinguales.6064

Ce document a été généré automatiquement le 14 février 2022.

\section{cc) $(1) \ominus$}

Multilinguales est mise à disposition selon les termes de la Licence Creative Commons Attribution Pas d'Utilisation Commerciale - Pas de Modification 4.0 International 


\title{
Une lecture de la reception du roman de langue kabyle (ou l'ungal)
}

\author{
A lecture on the reception of the berber (kabyle) novel
}

\author{
Nabila Sadi
}

1 La réception est une dimension inhérente à la nature même d'un genre littéraire. Elle, qui se définit comme "l'activité de lecture et d'interprétation des textes» (Kherdouci H., 2017 : 14), n'accueille pas l'œuvre de manière passive puisque c'est par la réception que cette dernière prend son plein sens. Le lecteur ne saurait être qu'un élément de la triade (auteur-œuvre-lecteur) comme le signale H. R. Jauss (2010), mais bien « une énergie qui contribue à faire l'histoire» (49), participant, de ce fait, pleinement à l'expérience littéraire et à la mouvance de l'horizon d'attente. Et formuler un discours autour du discours critique, c'est procéder à rebours de la pratique courante pour proposer une sorte de métadiscours dans lequel le discours critique est lui-même transformé en objet d'étude. Il s'agit, ici, de proposer une lecture de la réception de l'ungal qui se décline au niveau de la critique universitaire, des comptes rendus de lecture et quelques «mentions rapides dans les émissions de la radio kabyle " (Salhi M. A., 2011: 94). Mais il est surtout question d'historiciser les différentes positions des critiques, de les corréler à l'état du champ littéraire kabyle, à différents moments de son histoire pour observer la manière dont il a impacté les différentes lectures de l'ungal, et comment celles-ci, par ricochet, ont contribué au processus de légitimation et d'institutionnalisation de ce genre. Car comme le note P. Bourdieu (1998), le discours autour d'une œuvre

«n'est pas un simple adjuvent, destiné à en favoriser l'appréhension et

l'appréciation, mais un moment de la production de l'œuvre, de son sens et de sa valeur » (285).

\section{L'accueil d'un genre littéraire emergent : l'ungal}

2 La naissance officielle ${ }^{1}$ de l'ungal, au début des années 1980, n'a pas tardé à susciter l'intérêt de la critique universitaire puisque la première étude en date, celle de D. 
Abrous, s'aligne au rang des approches anthropo-littéraires en appréhendant ce genre dans une dynamique de passage de l'oralité à l'écriture (D. Abrous, 1989). Elle s'appuie sur les trois seuls textes romanesques attestés comme tels à cette époque-là (Asfel, Askuti et $F a f f a^{2}$ ) pour énumérer les points de continuité et de rupture que marque l'ungal par rapport à la tradition littéraire orale. Ceux-ci sont particulièrement discutés à partir du contexte socio-politique des années 1980 concomittant avec la montée des revedications identitaires en Kabylie dont l'ungal n'est que l'expression de ces mutations socio-culturelles, explique la même auteure. C'est pourquoi, la question de l'identité et de son impact sur ces trois ungal investit une large part de ce travail. L'usage des néologismes pour pallier à la suppression des emprunts à la langue arabe est, à ce propos, représenté comme un choix motivé de ces trois romanciers, témoignant ainsi d'un réel positionnement idéologique ${ }^{3}$. Ce contexte historique déteint, aussi, sur la thématique de ces trois romans qui reprennent, sous des formes variables, la question identitaire.

3 Le choix d'une approche anthropo-littéraire s'explique, à la fois, par la tendance «anthropologisante» des études portant sur la littérature kabyle jusque-là, notamment celles consacrées à la littérature orale mais aussi, par la nature et la vocation même de l'ungal dont la naissance officielle coïncide avec le contexte revendicatif des années 1980 . C'est pourquoi, la tendance des premières études portant sur l'ungal gravitait autour du contexte ${ }^{4}$ ayant vu émergé ce genre et de ses répercussions sur les reconfigurations génériques. L'ungal est, alors, amené à ne pas être interrogé dans sa singularité, mais au sein de la structure vaste de la littérature kabyle écrite comme il est notable dans une étude de D. Abrous (D. Abrous, 2004). Il est, plutôt, replacé dans une dynamique évolutive de cette dernière (intégrant d'autres genres comme tullist/nouvelle et amezgun/théâtre), contribuant, ainsi, à la production d'un champ notionnel visant à rendre compte de ces nouvelles réalités littéraires, à l'image de "néo-littérature/nouvelle littérature ", "littérature écrite " (Chaker S., 1992) ou encore "littérature émergente» (A. Ameziane, 2008: 131-137). Elles représentent autant d'appellations dont le but est de rendre compte du nouveau paysage littéraire kabyle et des inspirations et motivations (modernisme, quête identitaire, etc.) ayant pu impulser son émergence. Ces premières études donnent, ainsi, forme à un discours "sur " l'ungal correspondant -pour reprendre les termes de C. Moisan (1987)- à la "phase d'admission» d'un genre, qui est une sorte d'«introduction, d'un discours d'entrée, qui donne un droit d'entrer" (220). La prise en compte de ce contexte d'émergence est un préalable indispensable auquel M. A Salhi dédie d'innombrables textes dans lesquels il y traite l'ensemble des éléments déterminant l'existence de l'expression romanesque kabyle en interrogeant, entre autres, les conditions éditoriales et institutionnelles, le statut de la langue amazighe, la situation sociolinguistique, etc. (Salhi M. A., 2006, Salhi M. A., 2011). S. Chemakh (2010) y observe, également, une étape décisive :

« avant de mener des études thématiques ou d'entamer des analyses littéraires du roman, de la nouvelle ou de la poésie kabyle écrite, il semble nécessaire de décrire les conditions d'existence de cette néo-littérature » (163).

C'est du côté de l'écrivain qu'il place cette problématique en décrivant les conditions de production des différents genres constituant la littérature kabyle écrite en insistant sur la compétence linguistique, littéraire et la motivation sociale des auteurs de langue kabyle (Chemakh S., 2010). 
La question de l'édition se taille une place de choix au sein de ce discours d'entrée. Le romancier A. Mezdad, en incluant sa propre expérience dans l'écriture romanesque, initie le débat en posant le problème de l'écrivain de langue kabyle (A. Mezdad, 2001). Les difficultés liées à la production (publications à compte d'auteur) et à la réception (lectorat réduit) sont posées comme deux grands obstacles à la prospérité de l'ungal. De manière un peu plus ciblée, c'est l'expérience éditoriale du haut commissariat de l'amazighité et de son degré d'impact sur le champ littéraire algérien en tant qu'instance de légitimation de la production amazighe qui se retrouve interrogée dans une étude de A. Ameziane (Ameziane A., 2011). Bien que cette institution offre une opportunité aux auteurs de se faire publier en langue amazighe, cet auteur souligne "l'impact négatif » de sa politique éditoriale qui affiche parfois un manque de suivi par rapport aux ouvrages qu'elle publie. Il cite le cas de Bu-tqulhatin dont la réédition à compte d'auteur présente plusieurs anomalies qui le poussent à supposer que la commission de lecture "ne semble pas agir sur le contenu des ouvrages que le HCA édite» (Ameziane A., 2011 : 374). Ce discours d'entrée ouvre, ainsi, les portes à l'ungal par le biais d'un discours attestant des transformations littéraires ayant amené l'introduction de genres inédits, mais il se consacre, tout particulièrement, à son fonctionnement institutionnel au sein du champ littéraire algérien.

La pratique des comptes rendus de lecture entre, également, en résonnance avec la critique universitaire dans la mesure où elle intègre, à son tour, cette phase d'admission de l' ungal

, bien que par des stratégies divergentes. En effet, paradoxalement aux autres travaux de recherche sur l' ungal

, la pratique des comptes rendus de lecture est, souvent, réalisée en langue kabyle. Une stratégie qui identifie, non seulement, le lectorat visé par cette pratique, mais qui exprime, par la même occasion, la capacité de cette langue à produire non seulement une littérature écrite mais, aussi, à porter un discours sur cette même littérature. La question linguistique occupe, d'ailleurs, une place de choix au niveau de ces comptes rendus qui insistent, pratiquement tous, sur la facilité de lecture des ungal

abordés. C'est à cette occasion que K. Naït Zerrad évoque la fluidité de la langue usitée par D. Benaouf dans

Timlilit ntyermiwin

malgré les quelques néologismes qui la traversent (K. Nait Zerrad, 2001). Les auteurs de ces comptes rendus mettent, souvent, l'accent sur le degré de recours des romanciers aux néologismes dont l'usage démesuré peut être très contraignant au moment de la lecture. Toutefois, l'entreprise de certains romanciers est, parfois, saluée au regard du travail effectué sur la langue romanesque. En parlant de

Faffa, N. Abrous énumère les mérites de R. Aliche qui « n'a cessé de triturer et d'essorer la langue, la nouer et la pétrir pour rendre un univers sémantique intarissable » (N. Abrous, 2008). Le talent d'A. Mezdad, selon A. Hamidouche, réside dans la simplicité de la langue du roman

Tagrest Uryu 
, marquée par un retour constant aux archaïsmes de la langue kabyle (A. Hamidouche, 2000). Malgré cela, les difficultés que pose cette même langue pour ces romanciers qui n'ont eu l'occasion de la pratiquer que dans son usage oral, ne sont pas passées sous silence. C'est dans ce sens que M. A. Salhi reconnaît que la langue d'

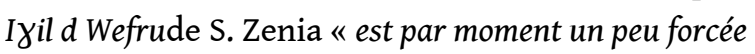

» (M. A. Salhi, 2003). Une conséquence presque naturelle d'une langue évoluant dans un milieu marqué par le plurilinguisme, ajoute le même auteur. Néanmoins, l'accent est majoritairement porté sur la lisibilité et l'accessibilité de certains

ungal

à la lecture, chose qu'affirment, par exemple, S. Chemakh et T. Ould Amar à propos de Salas d Nujade B. Tazaghart (Chemakh S., 2005, Ould Amar T., 2006).

A leurs premières manifestations, ces comptes rendus se consacrent rarement à la présentation seule des textes dont ils proposent une lecture mais se livrent aussi à des présentations générales de l'

ungal. En effet, en raison du problème de diffusion que connaissent les productions romanesques en langue kabyle (notamment à leurs débuts) et vu la publication de nombreux textes à l'extérieur du territoire national, il fallait, donc, pour l'essor de ce genre, attester de l'existence de ces textes en les replaçant dans leur « lieu naturel » (P. Bourdieu, 1998 : 276) dans lequel ils sont censés prendre place et être consommés, vu qu'aucune prise en charge institutionnelle n'œuvrait à leur promotion. En parlant des ungal publiés en France, M. A. Salhi (2006) écrit qu'il fut un temps où

«leurs auteurs ne sont reconnus comme romanciers que par l'information et la critique journalistiques ou l'information militante » (125).

C'est là que se mesure tout l'intérêt de la critique car comme le note P. Bourdieu (1998) :

«Le travail de fabrication matérielle n'est rien sans le travail de production de la valeur de l'objet fabriqué » (287).

C'est l'une des raisons qui font de ces lectures un lieu mobilisant plusieurs stratégies de valorisation par lesquelles certains auteurs vont jusqu'à niveler les romanciers kabyles à des écrivains de renommée internationale (tels M. Proust, W. Faulkner, A. Camus) comme il est possible de le noter dans certains textes consacrés à R. Aliche $(\mathrm{N}$. Abrous, 2012) ou à B. Tazaghart (T. Ould Amar, 2006). Le critique participe, ainsi,

«à faire la valeur de l'auteur qu'il défend par le seul fait de le porter à l'existence connue et reconnue, d'en assurer la publication [...] en lui offrant en garantie tout le capital symbolique qu'il a accumulé $»^{5}$ (Bourdieu P., 1998 : 287).

Visant à asseoir une certaine autorité à l'ungal

, cette pratique s'insère au sein de cette phase d'admission de l'ungal

. Ces stratégies de valorisation ainsi que la constante mise en relief de l'accessibilité de la langue de l' ungal

rend palpable certains aspects contraignants du champ littéraire dans lequel évoluait le genre. Prôner certains textes de l' 
ungal

comme très faciles et agréables à la lecture se dresse en réponse au problème accru de lectorat que connaissait ce genre. Une réalité que ne manque pas de noter D. Merrola qui observe que le problème de communication entre les auteurs de romans kabyles et leur public est beaucoup plus accru qu'il ne l'est pour le romancier de langue arabe ou française dans le milieu maghrébin, dont le lectorat est déjà assez réduit ( Merolla D., 2006 : 183). En effet, avec le passage à l'écrit, il fallait créer une habitude de lecture en langue kabyle qui n'est pas une pratique qui suit de manière « naturelle » le cours des choses mais bien une disposition qui, pour s'accomplir, nécessite la mobilisation d'un ensemble de facteurs comme la promotion et la médiatisation du livre amazighe, la prédisposition à la lecture en langue amazighe grâce à l'enseignement de cette langue, etc.

9 La réception de l'ungal diffère, ainsi, selon le type d'institution d'où elle émane. Les premières appréciations de cette nouvelle prise de position des auteurs (autrement dit, l'écriture d'ungal) ont été largement structurées en fonction des données du champ littéraire kabyle en rapport avec sa position au sein du champ littéraire dominant. Les premiers "réflexes » observés au niveau de la critique universitaire ainsi que dans la pratique des comptes rendus de lecture élaborent davantage un discours «sur » l'ungal. Ces derniers, s'adressant à un public plus élargi, concourent à offrir une visibilité à cette forme d'expression dont la majeure partie de ses productions était, pendant longtemps, assurée par des maisons d'édition internationales. Ce type de réception répondait plus directement aux exigences qu'imposait ce même champ littéraire qui était démuni de toutes les composantes nécessaires pour assurer la visibilité et la promotion de l'ungal (et de la littérature kabyle de manière générale). C'est pourquoi, à cette situation défavorable et contraignante, la réception (au niveau des comptes rendus) avait réagi positivement en louant les vertus des écritures de certains auteurs, optant par moments pour une stratégie de surenchère de certaines œuvres en les nivelant à celles d'auteurs de notoriété internationale, pour stimuler un lectorat qui était dépourvu de toute prédisposition à la consommation (lecture) en langue kabyle (statut non institutionnalisé de la langue amazighe, indisponibilité des ouvrages, etc). Pareillement dans la critique universitaire, de nature plus ésotérique puisque s'adressant davantage à ses pairs, elle affiche également, à ses débuts, un discours qui servait d'introduction à cette nouvelle forme d'expression dans l'espace culturel en insistant sur les conditions de son existence.

Par ailleurs, l'aspect et le contenu des comptes rendus ces dernières années, plus tournés vers la description de la poétique des ungal ${ }^{6}$ sont, d'ailleurs, une indication de nouvelles données en matière de lectorat. Dans un entretien réalisé il y a maintenant quelques années avec M. Kezzar, M. Zimu et S. Zenia, ce dernier observe le regain d'élan qu'a connu l'édition en berbère. Il affirme que :

«Si l'édition a pris plus d'essor, cela veut dire qu'il y a un lectorat, même relatif, mais il existe. Avant, les éditeurs réfléchissaient deux fois avant d'éditer un quelconque ouvrage, non seulement en tamazight mais aussi dans les autres langues » (S. Zenia et al., 2011).

En effet, nous assistons, présentement, à une vague de réédition d'ouvrages de littérature kabyle (dont plusieurs

ungal $^{7}$ ) notamment avec le concours des éditionsTira 
. L'épuisement de certains stocks de réédition (M. A. Salhi et A. Ameziane, 2017 : 326) nous invite à réévaluer la question du lectorat, notamment, depuis que l'enseignement de la langue amazighe s'est généralisé en Kabylie avec l'implantation de trois départements au niveau de cette dernière (

Tizi Ouzou,BejaïaetBouira

) et un autre département à Batna, formant, ainsi, des centaines de diplômés tous les ans, autrement dit, une prédisposition à la consommation (la lecture) du livre écrit en kabyle (tamazight).

\section{La problématique du renouvellement littéraire}

11 Certaines positions de la critique manifestent le souci de recherche d'une voie propre dans l'étude de l'ungal. Ainsi peut-on dire des nombreuses études insérant ce genre dans une dynamique évolutive de la littérature kabyle, misant sur le rapport de l'ungal à l'oralité traditionnelle pour pouvoir déceler les procédés du renouveau littéraire. L'objectif étant de déceler quelques mécanismes de la création littéraire qui passent par l'appropriation et la subversion du fond kabyle oral. Ce qui est souvent mis en exergue, sont les passerelles qui s'établissent entre ces deux pans de la littérature amazighe (la littérature écrite et la littérature orale) même lorsque la première s'en désolidarise totalement pour manifester une nette rupture vis-à-vis de la tradition orale. C'est dans le sillage de ces études que M. A. Salhi discute, à titre d'exemple, le recours constant des auteurs aux ressources de la littérature orale pour leur usage comme matrice essentielle à la composition de nouveaux textes (Salhi M. A, 2004). Ces narrations orales (le conte plus particulièrement) deviennent, par moments, le point de départ par lequel est discuté l'ungal par rapport au renouvellement qu'il opère d'un point de vue narratif et thématique (Berdous N., 2001).

Cette tendance n'est pas stérile et ne se résume pas à une simple recension des motifs de la littérature orale dans l'ungal. A. Ameziane va se consacrer longtemps à cette problématique. D'abord, dans Les formes littéraires traditionnelles dans le roman kabyle où il repère, non seulement, la présence de textes oraux dans l'ungal mais formule une réflexion sur la manière dont ils sont transformés en contexte d'écriture (Ameziane A., 2002). Intéressé fortement par cette question, il élargit, ensuite, sa problématique dans sa thèse de doctorat (Ameziane A., 2008) en se penchant sur la mise en texte de ces formes traditionnelles et les différentes fonctions et significations "nouvelles » dont elles se dotent une fois transposées dans d'autres genres relevant de l'écrit. L'auteur parvient à rendre compte de quelques aspects du renouvellement de la littérature kabyle s'élaborant sur la base de la réactualisation de motifs traditionnels, de la parodie ou de la transformation totale d'un genre littéraire oral.

13 Le rapport ungal/oralité jouit, ainsi, d'un grand intérêt au niveau de la critique littéraire kabyle. Cette tendance est conditionnée par la représentation qu'a été faite de ce genre, considéré comme " une forme de passage à l'écrit ». Au moment où certains chercheurs clament l'essoufflement des genres oraux ${ }^{8}$, et que parallèlement, le champ littéraire kabyle accueille d'autres formes littéraires qui se construisent et y prennent place, étudier le lien qu'entretient l'ungal avec ses prédécesseurs constitue un passage presque obligatoire dans le parcours de la critique romanesque kabyle. Cette dernière ne faisant, ainsi, que suivre l'élan de l'évolution de la littérature kabyle mais en saisissant cette problématique comme moyen permettant de comprendre l'un des 
mécanismes de la création et du renouvellement littéraires. Cette approche, traduit, toutefois -ne serait-ce que de manière tacite- une manière de concevoir les genres littéraires «nouveaux » et plus précisément les genres écrits dans un processus et une dynamique intra-littéraires. De surcroit, démontrer comment les genres oraux sont transformés et réhabilités dans l'ungal dessine un nouveau rapport à ces genres et à leur traitement, hors de leur contexte naturel d'existence et projette, par la même occasion, une conception de l'ungal dans une perspective intra-générique et intraculturelle.

\section{L'impact des approches immanentistes}

14 À partir des années 2000, le discours critique s'engage dans une nouvelle voie avec une tendance immanente très prononcée au niveau des études consacrées à l'ungal. Celles-ci s'attèlent à la description de la forme et du discours « de » l'ungal par l'exposition de plusieurs caractéristiques de sa poétique, répondant ainsi à des exigences d'ordre interne. Cette orientation est réalisée, presque exclusivement, par les trois départements de langue et culture amazighes (Tizi Ouzou, Bejaïa, et plus récemment Bouira) ${ }^{9}$. Pendant longtemps, la tendance générale fut à l'approche sémiotique. Elle se lit dans l'étude de F. Achili qui, en prenant pour objet la triologie de A. Mezdad (I d d wass, Tagrest uryu et Ass-nni) tente d'y mettre en relief la structure narrative et discursive (Achili, 2015). Dans la même lignée, O. Bourai, en faisant appel au même type d'approche (dite sémiotique), se propose d'exposer l'organisation du discours du roman Asfel sur le plan actoriel, spatial et temporel tout en s'attachant à relever les principales oppositions sémantiques mises en place par le texte (Bourai O., 2007). N. Berdous interroge, également, le volet narratif (techniques des points de vue, rapport narrateur/narrataire) en relation avec la transition opérée de l'oral vers l'écrit. Elle envisage la thématique de ces ungal comme l'un des plus grands aspects du renouveau littéraire et affirme que les auteurs se sont beaucoup plus préoccupés de celui-ci, négligeant le côté narratif, qu'elle juge très proche du style oral. Les techniques narratives sont également explorées dans l'étude de F. Hacid (Hacid F., 2008) qui aborde la problématique de la création littéraire kabyle à travers l'étude de la structure et de la temporalité narratives dans le roman I $\underset{d}{d} d$ wass d'A. Mezdad. Dans la perspective d'une combinatoire, celle de la sémio-anthropologie, nous avons consacré une étude à la question de l'expression de l'identité dans le roman Tafrara de S. Zenia (Sadi N., 2011). L'identité n'y est plus envisagée comme simple contenu mais en tant que procédé de création littéraire intervenant à différents niveaux de la poétique de l'ungal, notamment au niveau du narrateur, du personnage et de l'espace.

15 Il est d'ores et déjà possible d'observer que les textes de R. Aliche, d'A. Mezdad et de S. Zenia occupent le pôle position en matière de corpus soumis à l'étude, ce qui contribue non seulement à appuyer l'autorité de ces auteurs mais sert, également, à mettre en lumière quelques caractéristiques de leur poétique. Ainsi en est-t-il de la première étude entièrement consacrée au personnage, à savoir celle de $\mathrm{N}$. Bellal qui part du postulat que les personnages mis en scènes par A. Mezdad constituent des archétypes. En joignant l'analyse sémiologique à l'approche sociologique, il explicite les différents procédés usités par le romancier dans la constitution de ses personnages et tente d'en fructifier la valeur idéologique en présentant la vision du monde de ce romancier (Bellal N., 2012). Un autre texte du même romancier s'adonne, aussi, à une étude de 
type stylistique. K. Hireche aborde le discours féminin dans le roman Ass-nni d'A. Mezdad en prêtant attention à la manière dont l'auteur fait parler les personnages féminins en fonction de leur profil, de leur espace d'énonciation et des contenus qu'investissent leurs propos (discours sur les droits de la femme, croyances, conditions de vie, etc.) (Hireche K., 2017).

Bien qu'ils aient mis en avant l'insertion de l'ungal au sein d'un champ littéraire en transformation, et l'ont représenté comme le véhicule de questionnements idéologiques majeurs (l'identité principalement), la critique émanant des institutions universitaires investit, notamment à partir des années 2000, la problématique de la création littéraire (en se consacrant à la question du renouveau littéraire). Mais ceux-ci dessinent, également, quelques grandes lignes de la poétique de ce genre en optant pour des approches immanentistes bien que certaines d'entre-elles réduisent un temps soit peu l'ungal à un "simple corpus d'application» (Salhi M. A. et Sadi N., 2016:31). En effet, l'application de ces grilles d'analyse déjà toutes préconçues - quoique prises comme un ensemble, elles esquissent quelques lignes de la poétique de l'ungal - régente toutefois les dimensions de l'œuvre, désamorce sa charge symbolique et la détache de son contexte qui façonne grandement les textualités et les conceptions qu'engendre ce genre. Néanmoins, ce revirement de la critique, en basculant d'un discours "sur " l'ungal vers un discours « de » l'ungal, n'est dû, en grande partie, qu'à l'entrée en jeu de nouveaux paramètres comme l'institutionnalisation de la langue amazighe, son intégration dans l'enseignement scolaire, la formation des premiers diplômés en langue et culture amazighes, etc.

\section{La codification de l'ungal}

Comme il a été noté plus haut, les années 2000 représentent un marquage important dans l'étude de la réception de l'ungal. Elles inaugurent les prémisses des interrogations autour de la codification de ce dernier dont l'institutionnalisation ne s'achemine pas de façon aisée. Cette phase de codification consiste à déconstruire l'ungal en un ensemble de codes, de règles, de lois, permettant d'identifier les textes se rattachant à cette même catégorie. Le soucis premier a été de délimiter le corpus représentatif de ce genre, ce à quoi s'est consacré $\mathrm{M}$. A. Salhi qui place l'élément linguistique, en l'occurrence la langue kabyle, et l'attribution de la mention d'« ungal/ roman » que ce soit par l'auteur lui-même, l'éditeur ou le critique, comme premiers éléments distinctifs de ce genre (Salhi M. A., 2011: 82). Après avoir marqué cette identité linguistique, le même auteur procède à la mise en comparaison des genres dits « nouveaux » face à leurs prédécesseurs, à savoir la littérature kabyle traditionnelle. En effet, l'identité de l'ungal s'élabore souvent en comparaison à la tradition orale, qui n'admettait pas plusieurs des caractéristiques de ce genre. M. A. Salhi (2000) note, à ce propos, que ces nouveaux genres

«se distinguent fortement des textes traditionnels par leur langue, leur

thématique, leurs styles et leurs structures » (249).

18 A la même période (début des années 2000) le même type de réflexion s'entend chez A. Mezdad $^{10}$, mais à propos d'un texte bien particulier, Lwali $n$ udrar de B. At Ali. Il voit dans l'implication de B. At Ali dans ses écrits et la nomination des personnages des signes de "pré-roman » (Mezdad A., 2000). La définition de l'ungal commence, alors, à prendre place, engendrant des réflexions sur sa généricité ${ }^{11}$. En faisant reposer l'ungal 
sur trois dimensions essentielles (les actions, les personnages et l'intrigue), sans lesquelles il n'y aurait de roman, A. Mezdad définit ce genre comme :

Une œuvre en prose généralement assez longue, dont l'intérêt est dans la narration d'aventures, l'étude de mœurs ou de caractères, l'analyse de sentiments ou de passions, la représentation objective ou subjective du réel. C'est une œuvre qui doit comporter une action et une intrigue, des personnages et quelques connaissances générales ou spécifiques pour le côté documentaire. (Mezdad A., 2000).

Le roman s'érige, ainsi, en une sorte de passerelle entre la réalité et la fiction, en une forme intermédiaire entre le réel et cette activité créatrice qui puise du premier en le « symbolisant ${ }^{12}$. Une idée que reprend, également, A. Ameziane :

" écrire un roman est plus exigeant qu'écrire un simple récit car alors que le second se contente de focaliser sur les événements, le premier exige de rendre compte de la complexité du monde » (Ameziane A., 2016).

L'ungal commençait, ainsi, à être de plus en plus pensé en tant que catégorie générique. Et l'une des conséquences de l'émergence de ce débat s'est manifesté en termes d'assignation générique. Celle-ci s'est observée notamment dans la chaîne de réception qui s'est constituée autour de l'œuvre de B. At Ali dont la plupart des textes ne véhiculaient nulle indication générique ${ }^{13}$. Et le texte Lwali $n$ udrar, tout particulièrement, a été sans cesse recontextualisé en vue de nouvelles relectures ayant eu un impact direct sur sa reconfiguration générique, le faisant basculer de la catégorie de conte, à nouvelle et enfin, au roman (Mohand Saidi S., Sadi N., 2016). Ces classifications génériques variables ayant accompagné ces relectures successives sont autant d'indications patentes de l'évolution de la réception et de l'horizon d'attente. Sous l'effet de ces nouvelle données, de la croissance des publications romanesques en langue kabyle et de la familiarisation de la critique avec l'ungal qui était, désormais, une formulation générique " attendue », les critères ayant été usités pour la réception de ce texte en tant que "conte» ont eux-même été sous l'emprise de cette mouvance. N'ayant plus la même connotation générique d'antan ${ }^{14}$ (Sadi N., 2019: 108), ils sont, dorénavant, représentés comme des éléments puisés de la tradition orale mais dont le caractère subversif les engage au service de nouvelles esthétiques, dont l'ungal (Ameziane A., $2008: 100$ ).

20 Mais en dépit de la variété de la production de B. At Ali et de sa profusion, cette réception phénoménale n'a été rendue possible que dans la mesure où son œuvre représentait en soi un enjeu. La période à laquelle elle fut produite (entre1945-1946) est l'un des facteurs décisifs. Recontextualiser l'œuvre de B. At Ali dans le champ du présent pour d'innombrables relectures sert, non seulement, à élaborer un marquage temporel inaugurant une mutation au sein du système littéraire kabyle (naissance de la littérature kabyle écrite) mais il représente, aussi, un enjeu symbolique consistant à «faire date» (Bourdieu P., 1998 : 261) dans le champ littéraire algérien en attestant d'une prise de position anciennement établie, plus ou moins concomitantes des premières prises de position des littératures écrites dominantes (d'expression arabe et française) au sein du champ littéraire algérien. En effet, "l'ancienneté est un élément déterminant du capital littéraire » (Casanova P.,2008 : 33). Et au regard de l'évolution du champ littéraire kabyle à la périphérie du champ littéraire algérien dominant (détenteur du capital économique et politique), l'exhibition du capital littéraire et culturel par la répertoriation d'un texte ancien qui s'est canonisé au fil des réceptions, est, aussi, une stratégie d'acquésition du capital symbolique, une manière de rejoindre ces luttes symboliques qui marquent les champs littéraires. 


\section{La légitimation de l'ungal}

21 Par discours de légitimation, il faut entendre tout processus de validation, de reconnaissance d'une œuvre ( un texte, une catégorie de textes, un genre) émanant d'une institution (université, prix littéraire, etc.) détentrice d'un capital symbolique lui permettant de faire autorité et de consacrer la valeur littéraire, esthétique, générique de cette œuvre qu'elle se propose de discuter. Le processus de légitimation peut être, alors, entendu comme

«le discours des institutions qui concernent les sélections faites en vertu d'accord

(ou de désaccord) aux règles, aux coutumes, aux normes établies » (Moisan C., 1987 :

220).

De manière plus schématique, le discours de légitimation représente tout discours critique qui consacre l'œuvre comme étant « sérieuse ». Par ricochet, le procédé de ce même discours peut se manifester par un processus de "dé-légitimation » qui se matérialise par la reconsidération de cette même valeur littéraire, esthétique ou générique propre à une œuvre singulière.

C'est autour de la question des contiguités génériques -de plus en plus prégnante ces dernières années- que s'énonce ce discours de légitimation autour de l'ungal. Cette interrogation est une problématique de toutes les littératures. Le débat qui l'anime émane, souvent, d'une opposition entre le discours des critiques, de nature plus normatif, ne trouvant pas toujours échos dans les réalisations des auteurs qui ne voient pas forcément dans la rigidité des critères que propose ce discours un rouage essentielle à leurs productions. Pour le cas de la littérature kabyle, la dénomination générique d'ungal est largement employée dans la désignation des productions littéraires écrites en langue kabyle, mais elle réunit parfois des écritures d'apparence dissemblables. Alors qu'une première constante reprend des critères largement partagés par le roman occidental, la seconde entraîne des perspectives esthétiques autres en empruntant à d'autres genres (comme tullist/nouvelle, tamacahut/conte) leurs techniques. La production romanesque en langue kabyle ne présente, donc, pas un corpus homogène en matière de généricité. Et de ce fait, la définition de l'ungal engage deux voies divergentes au niveau de la critique. La première, plus normative, mobilise des références cadrant avec la conception occidentale du roman, autant sur le plan formel que du point de vue de l'élaboration de l'histoire. Ainsi, N. Aït Ouali (2015) stipule que :

Le roman ou la nouvelle sont des produits d'une écriture créative qui sollicite l'imaginaire et le rêve. Refuser le monde dans lequel on vit et/ou dénoncer ce qui s'y passe ne suffisent pas pour faire une œuvre littéraire. Il faut rêver et imaginer un autre monde, différent, possible ou utopique. C'est l'universalité de l'écriture qui permettra au lecteur de partager ce rêve, ne serait-ce que le temps de la lecture. $(08)^{15}$.

Cette conception de l'histoire non exemplifiée par certains ungal, conjuguée à la longueur de certains textes jugés trop courts, sont interprétés comme un facteur ayant engendré " une véritable confusion des genres dans la littérature kabyle d'expression amazighe » (Ait Ouali N., 2015 : 08). Mais cette « universalité de l'écriture » qu'évoque $\mathrm{N}$. Aït Ouali, n'est en fait que la manifestation des catégories littéraires occidentales (voire françaises) s'étant imposées sur le monde littéraire par la forte domination de l'espace auquel elles appartiennent (Casanova P., 2008 : XV). La globalisation du modèle 
romanesque comme modèle théorique ne peut se faire que par la relativisation de certains « réflexes spontanés » et quelques " habitudes littéraires » qui verraient dans certaines «déviations " génériques une hérésie ( Casanova P., 2008: XIV). Il faudrait plutôt songer -comme le note $\mathrm{P}$. Casanova à propos de l'élaboration de son modèle explicatif du fonctionnement de la république mondiale des lettres- à mettre en place des outils qui puissent reconnaître une légitimité à d'autres littératures qui ne fonctionnent souvent pas selon la même logique de cet espace dominant. L'esprit de cette réflexion se lit chez $\mathrm{M}$. A. Salhi (seconde orientation de la critique) où une nette évolution se dessine dans son traitement de cette problématique. Après avoir affiché des interrogations à l'égard de l'identité générique de quelques textes et de leur éventuelle lecture en tant que nouvelles/tullisin (Salhi M. A., 2011 : 92-93) ${ }^{16}$, il tranche sur la question dans une étude inédite, notamment en matière d'orientation théorique dans le traitement de ce type de manifestations.

Il affirme que ces contiguïtés génériques ne représentent « ni une confusion des genres, $n i$ une anomalie éditoriale» mais bien une conséquence naturelle des cultures en transformation (Salhi M. A., texte inédit). Des questionnements s'entendent, également, chez Z. Meksem à propos de la variation des identifications génériques, vacillant entre ungal et tullist, qu'on lui avait proposé pour son texte Tabrat $n$ uzekka. Bien que la première de couverture indique clairement son identité d'ungal, Z. Meksem (2015) choisit dans son mot d'auteur au début du texte, de laisser une ouverture à son éventuelle lecture en tant que tullist : "adris-a nezmer, ahat, ad t-nwali d ungal acku deg watas $n$ tulmisin-is yeffel $i$ tullist; nezmer ad $t$-nwali d tullist acku wezzil ibedd $s$ tuget $\gamma e f$ umazrar $n$ tedyanin ${ }^{17} »(06)$. La présence de ce type d'interrogation indique au moins un élément: Si l'on connait énormément de choses sur le roman, on en sait encore très peu sur l'identité de l'ungal.

Cette difficulté de se départir de l'influence de l'école étrangère a irrémédiablement marqué les appréciations de certains critiques en matière de légitimation du corpus représentatif de l'ungal. Comme celui-ci ne s'engage pas dans une voie homogène, notamment du point de vue de sa conception, les spécificités de ce corpus, fortement imprégné de l'actualité géo-politique de l'espace auquel il appartient, ont parfois été interprétées comme des insuffisances. La naissance d'un système littéraire écrit en langue kabyle dont les genres le composant y ont, plus ou moins, pris place à la même période, a fait que les frontières ont parfois été traversées pour emprunter certains aspects propres à un genre ou à un autre (Sadi N., 2019). Si les tullisin/nouvelles ${ }^{18}$ ayant adopté une conception se rapprochant de l'ungal, manifestant une poétique du romanesque, n'ont pas été confrontées au discrédit de la réception, les "promiscuités » de l'ungal avec d'autres genres comme tullist ou tamacahut/conte ont mis un point d'orgue au niveau de la critique. À croire que le roman s'enferme dans une servilité telle qu'il en devient fortement canonisé, que tout dépassement esthétique, poétique, est interprété comme une défaillance. Or, le choix de ces conceptions "disparates", puisant de la prose orale, de tullist, etc., renseigne sur les significations de l'acte d'écrire un ungal dont la valeur symbolique était, entre autres, de porter une langue orale dans un genre "écrit», amenant les propriétés significatives de la mention « ungal » à s'élargir dans une extension de sens qui l'assimile dans certaines de ses réalisations à un « livre » écrit en langue kabyle, à un projet d'écriture dans une langue qui était jusque-là orale (Sadi N., 2019:173). Et toute la difficulté de penser ces «promiscuités» tient dans la position méthodologique à adopter. Repenser ces 
productions selon la logique du champ littéraire dans lequel elles sont réalisées, comme le suggère $\mathrm{P}$. Bourdieu dans sa théorie des champs, est une voie féconde dans l'étude des genres littéraires.

Et dans cette perspective, la prise en compte de la singularité de ces conceptions en tant que "contiguïtés génériques", pour reprendre la dénomination de M. A. Salhi, représente en soi une position infiniment singulière au sein de la critique car elle s'attache à rendre explicite le fonctionnement d'une littérature émergente en élaborant de nouveaux codes de lecture et d'évaluation d'un système littéraire en transformation dont ces contiguités génériques en sont la conséquence directe, selon le même auteur. Et comme tout champ littéraire est un champ de lutte (Bourdieu P., 1998 : 263), ces productions non canonisées exprimant ces contiguïtés ne sont en fait qu'une réponse -parmi d'autres - que l'on avait oublié d'interroger par rapport à la problématique à laquelle elle réagissait, à savoir la production d'une littérature écrite en langue kabyle (Sadi N., 2019 : 316).

La réception intéragit, donc, bel et bien avec le champ littéraire et l'espace social dans lequel celui-ci évolue. Les conditions défavorables qui ont longtemps couvé sur les productions littéraires écrites en langue kabyle (absence de prise en charge éditoriale des textes, publication à compte d'auteurs, invisibilité des textes, etc.) ont amené la réception à s'organiser pour pallier ces conditions contraignantes. Elle a, ainsi, contribué à faire émerger cette forme d'expression en lui consacrant études et comptes rendus, contribuant à attester de son existence et à décrire les conditions dans lesquelles elle évolue. Ces conditions ont amené la réception de l'ungal à s'adapter à ces contraintes « externes » en déployant plusieurs stratégies de valorisation (notamment au niveau des comptes rendus de lecture) visant à asseoir une certaine autorité à l'ungal et à œuvrer à l'amélioration des conditions de son lectorat. Et dès lors que le champ littéraire s'est vu attribué de nouvelles données (ouvertures des départements de langue et culture amazighes, enseignement et institutionnalisation de la langue amazighe), une nouvelle orientation de la critique de l'ungal prend place pour répondre à des interrogations plus « internes » aux textes. C'est ainsi qu'à partir des années 2000, s'élève tout un discours de légitimation de cette forme générique (centré autour de la poétique des textes) s'accompagnant, également, d'une orientation à caractère plus prescriptif, s'interrogeant à propos de la codification de l'ungal et de l'ambigüité de certaines assignations génériques, perçues par certains critiques comme aberrantes. Entre prescription et réflexions sur le corpus de l'ungal, ces discours dépendent grandement des positionnements méthodologiques de ces critiques et du système de références (souvent imprégnés des canons de la littérature occidentale) à partir duquel ils discutent les textualités de ce nouveau genre, encore en construction.

\section{BIBLIOGRAPHIE}

ABROUS, Dahbia, La production romanesque kabyle : une expérience de passage à l'écrit, Chaker, Salem (dir.), DEA, Université de Provence, 1989. 
ABROUS, Dahbia, «La littérature kabyle », Chaker, Salem (dir.), Encyclopédie berbère, $\mathrm{n}^{\circ} \mathrm{XXVI}$, Edisud, Aix en Provence, 2004, pp. 4067-4074.

ABROUS, Nacira, «Pensée pour Rachid Aliche, Auteur romancier d'expression kabyle. Encore un rempart qui tombe », Mars 2008. Disponible sur : [URL] : http:// www.kabyle.net/ Pensée-pourRachid-Alliche-Auteur, 3811.

ABROUS, Nacira, « Rachid Aliche : Șṣaba teğğa-d tayeḍ », La Dépêche de Kabylie, mars 2012, Disponible sur : [URL] : http://www.depechedekabylie.com/ddk-tamazight/106630-rachid-alicheaba-tea-d-taye.html.

AIT OUALI, Nasserdine, L'écriture romanesque kabyle d'expression berbère, l'Odyssée, Tizi Ouzou, 2015.

AMEZIANE, Amar, Les formes traditionnelles dans le roman kabyle : du genre au procédé, Bounfour, Abdellah (dir.), DEA, Inalco, 2002.

AMEZIANE, Amar, Tradition et renouvellement dans la littérature kabyle, Bounfour, Abdellah (dir.), thèse de Doctorat, Inalco, 2008.

AMEZIANE, Amar, « La littérature kabyle dans l'expérience éditoriale du HCA. Quelques notes exploratoires », Studi Africanistici, Naples, 2011, pp. 369-375.

AMEZIANE, Amar, « Une lecture de Yiwen wass deg tefsut, roman d'Amar Mezdad ». Disponible sur : [URL] http://www.ayamun.com/Janvier2016.htm\#Etude.

BELLAL, Hakima, « La question du genre entre le manuscrit de Belaïd At-Ali et son édition. Regard sur trois textes Tafunast igujilen, Lexdubegga et Sut taddart », Salhi, Mohand Akli (dir.), Iles dimesli, $\mathrm{n}^{\circ}$ 8, Tizi Ouzou, 2016, pp. 34-42.

BELLAL, Noureddine, Étude du personnage en tant que catégorie textuelle, dans les romans kabyles d'Amer Mezdad, Bouamara, Kamel (dir.), mémoire de magister, université Abderrahmane Mira de Bejaïa, $2011 / 2012$.

BOURAI, Ourdia, Asfel, étude narrative et discursive, Dourari, Abderrezak (dir.), mémoire de magister, Ummto, 2007.

BOURDIEU, Pierre, Les règles de l'art. Genèse et structure du champ littéraire, Seuil, Paris, 1998.

Casanova, Pascale, La République mondiale des lettres, Seuil, Paris, 2008.

CHAKER, Salem, «La naissance d'une littérature écrite. Le cas berbère (Kabylie) », Bulletin des Études Africaines de l'INALCO, n 17-18, 1992, pp. 7-21.

CHEMAKH, Saïd, « Salas d Nuja ou l'amour possible », Mars 2005. Disponible sur : [URL]:http:// www. tamazgha/fr//Salas-et-Nuja-ou-l-amour-possible, 1267, html.

CHEMAKH, Saïd, « Les conditions de production de la néo-littérature kabyle », Asinag, n 4-5, Ircam, Rabat, 2010, pp. 163-168.

HACID, Farida, « Amar Mezdad, tettḍilli-d ur d-tkeččem : ungal [roman], Ayamun, Bgayet, 2014, 210p. ", Di Tolla, Anna Maria (dir.), Langues et Littératures berbères : Développement et standardisatio. Quaderni di Studi Berberi e Libico-berberi, Naples, 2014, pp. 171-173.

HADDADOU, Mohand Akli, Dictionnaire des racines berbères communes, Alger, HCA, 2006/2007.

HAMIDOUCHE, Abdelkrim, « Tagrest urghu (hivers brûlant), un grand roman en Tamazight", l'Hebdo $n$ Tmurt, $n^{\circ}$ 23, Août 2000. Disponible sur : [URL] http://www.ayamun.com/ Novembre2000.htm\#article. 


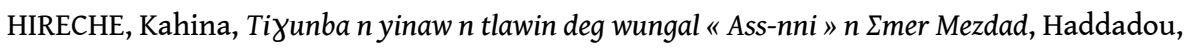
Mohand Akli (dir.), mémoire de magister, Ummto, 2017.

JAUSS, Hans Robert, Esthétique de la réception, Gallimard, Paris, 2010.

KHERDOUCI, Hassina, La chanteuse kabyle, une voix et une voie, La pensée, Tizi Ouzou, 2017.

LOIKKANEN, Sinikka, Vocabulaire du roman kabyle (étude lexico-statistique), Chaker, Salem (dir.), DEA, 1998.

MEKSEM, Zahir, Tabrat $n$ uzekka, Tira, Béjaia, 2015.

MEROLLA, Daniella, De l'art de la narration tamazight (berbère), état des lieux et perspectives, Peeters, Paris/Louvain, 2006.

MEZDAD, Amar « Vie et œuvre de Belaid At-Aâli, écrivain de Kabylie », 2000. Disponible sur : [URL] :http://www.ayamun.com/Mai2000.htm.

MEZDAD, Amar, « Perspectives dans l'édition du roman berbère ", Ayamun, n 9, Novembre 2001. Disponible sur : [URL] . URL : www.ayamun.com/Novembre2001.htm..

MOHAND SAIDI, Saïda et SADI Nabila, « Histoire de la réception des textes de Belaïd Ait Ali », SALHI Mohand Akli (dir.), Iles d imesli, n 8, Ummto, Tizi Ouzou, 2016, pp. 51-59. Disponible sur : [URL] : file :///C :/Users/DELL/Downloads/1474-5349-1-PB \%20(3).pdf.

MOISAN, Clément, Qu'est-ce que l'histoire littéraire ?, Paris, PUF, 1987.

NAIT ZERRAD, Kamel, «Timlilit n t\$ermiwin, s\$ur Djamel Benaouf », Ayamun, 2001. Disponible sur : [URL] : http://www.ayamun.com/Librairie.htm.

SADI, Nabila, L'expression de l'identité dans le roman Tafrara de Salem Zenia, Djellaoui, Mohamed (dir.), mémoire de magister, Ummto, 2011.

SADI, Nabila, « Brahim Tazaghart, Inig Aneggaru : ungal [roman] », Di Tolla, Anna Maria (dir.), Langues et Littérature berbères : Développement et standardisatio, Quaderni di Studi Berberi e Libicoberberi, Naples, 2014, pp. 175-178.

SADI, Nabila, Problématique de l'écriture romanesque en « kabyle », thèse de doctorat, Salhi, Mohand Akli (dir.), Ummto, 2019.

SALHI, Mohand Akli, « Les voies de modernisation de la prose kabyle », Actes du colloque international. Tamazight face aux défis de la modernité, Boumerdès, 2000, pp. 244-251.

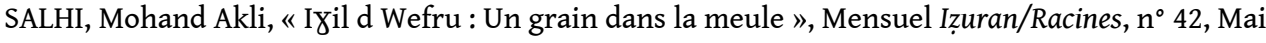
2003.

SALHI, Mohand Akli, «La nouvelle littérature kabyle et ses rapports à l'oralité traditionnelle ", Kich, Aziz (dir.), La littérature amazighe : oralité et écriture, spécificités et perspectives, Actes du colloque international, 2004, pp. 103-121.

SALHI, Mohand Akli, « Regard sur les conditions d'existence du roman kabyle », Studi Magrebini, Naples, vol. IV, 2006, pp. 121-127

SALHI, Mohand Akli, Études de littérature kabyle, Enag, Alger, 2011.

SALHI, Mohand Akli et Sadi, Nabila, «Le Roman Maghrébin En Berbère », Contemporary French and Francophone Studies, Irlande, vol. 20, 2016, pp. 27-36.

SALHI, Mohand Akli, Asegzawal amežzyan n tsekla, l’Odyssée, Tizi Ouzou, 2017. 
SALHI, Mohand Akli et Ameziane, Amar, «Le livre littéraire kabyle : édition et éditeurs », Littératures en langues africaines. Production et diffusion, Karthala, Paris, 2017, pp. 315-330.

SALHI, Mohand Akli, « Texte inédit », à paraître.

SCHAEFFER, Jean-Marie, « Du texte au genre. Notes sur la problématique générique », Genette, Gérard et Todorov, Tzvetan (dir.), Théorie des genres, Seuil, Paris, 1986, pp. 179-205.

OULD AMAR, Taher, «Quand les mots simples parlent de la vie », La dépêche de Kabylie, [en ligne]. URL : http:// www.bgayet.net/Salas-d-Nuja.html.

ZENIA, Salem, Zimu, Mourad et Kezzar, Meziane, « Écrire en kabyle aujourd'hui pour exister demain », propos recueillis par LATEB Azzedine, La Tribune, 2011. Disponible sur : [URL] http:// www.djazairess.com/fr/latribune/50459.

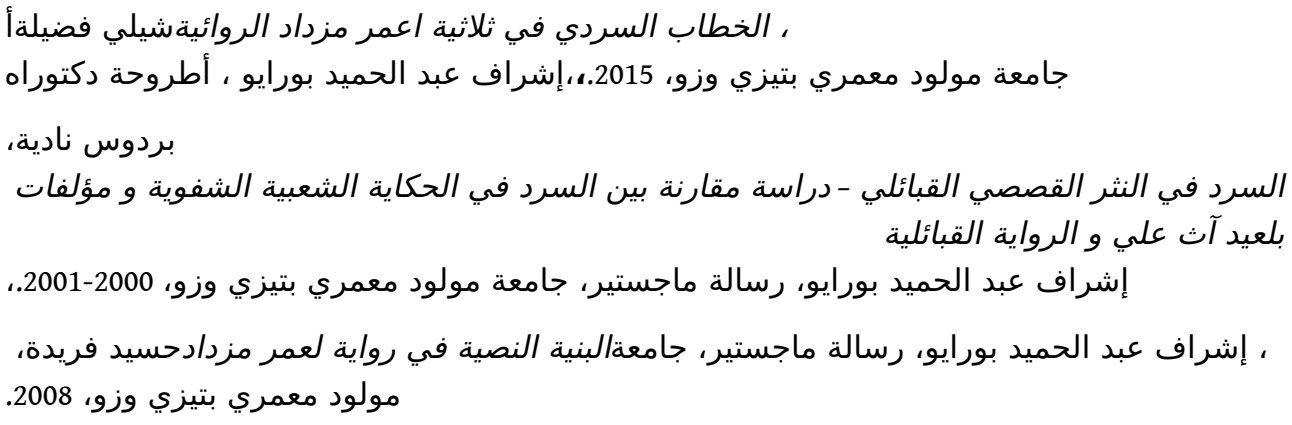

\section{NOTES}

1. Le premier texte portant la mention paratextuelle de "ungal» en première page de couverture est le roman Asfel de Rachid Aliche, publié en 1981. Néanmoins, la critique atteste, présentement, le texte Lwali $n$ udrar de Bélaid At Ali, écrit entre 1945-1946, comme le premier ungal/roman de langue kabyle.

2. Faffa (1986) de R. Aliche et Askuti (1983) de S. Sadi.

3. L'analyse du lexique de six ungal dans une étude de S. Loikkanen, soutenue à l'institut des langues et cultures orientales (INALCO), corrobore les résultats présentés par D. Abrous à propos de la prédominance des néologismes et de la suppression des emprunts à l'arabe au sein des premiers textes romanesques kabyles (Loikkanen S., 1998).

4. Des études plus récentes continuent encore à interroger cet aspect de la littérature kabyle écrite. Cela peut s'appliquer à l'étude de la narration en berbère de D. Merrola où elle interroge l'ungal, entre autres, en corrélation avec le contexte socio-historique des premières publications romanesques (D. Merolla, 2006).

5. L'impact de ce capital symbolique en termes de valorisation et de légitimation est variable selon le profil et la notoriété des auteurs de cette critique. L'étude de leur profil -qui serait sans aucun doute intéressante- gagnerait davantage à intégrer parmi ses interrogations la question de l'activité préfacielle. À titre d'exemple, les préfaces de deux textes (Asfel et Askuti), parmi les premiers ungal, ont été assurées par M. Mammeri dont la notoriété contribue naturellement à faire autorité.

6. Nous pensons, notamment, aux comptes rendus de M. A. Salhi (2003), de N. Sadi (2014), de F. Hacid (2014), d'A. Ameziane (2016), etc.

7. C'est le cas des ungal d'A. Mezdad, de Tafrara et Iyil d Wefru de S. Zenia, de Timlilit $n$ tyermiwin de D. Benaouf, de Askuti de S. Sadi, etc. 
8. C'est le cas d'A. Ameziane qui insiste sur l'essoufflement progressif de la littérature kabyle orale au profit d'une littérature écrite s'exprimant par le biais de nouvelles voies (Ameziane A., 2008).

9. Il s'agit, notamment, de mémoires de magister au niveau des départements de Bejaïa et de Tizi Ouzou, et de mémoires de master au niveau du département de Bouira.

10. A. Mezdad est l'auteur de plusieurs romans, d'un recueil de nouvelles et d'un recueil de poésie.

11. J.-M. Schaeffer propose de distinguer entre «genre » et " généricité ». Alors que le premier est une catégorie de lecture, dotée d'une composante prescriptive, agissant selon une fonction de classification, la généricité traduit une relation d'appartenance dans toute sa dynamique et est, par définition « une composante textuelle » susceptible d'être transformée et modulée (Schaeffer J.-M., 1986).

12. La racine «NGL», du mot "ungal», est pan-berbère. Elle engage deux significations divergentes. La première est relative à la «noirceur» (Haddadou M. A., 2006/2007 : 142). La seconde, quant à elle, reprend la sigification de "symbole», de "paroles symboliques ", de " paroles à sens caché ». ( Salhi M. A., 2012 : 98)

13. Voir à propos de la question du genre dans l'œuvre de B. At Ali, l'étude de H. Bellal ( Bellal H., 2016)

14. Parmi ces éléments, l'intitulé du premier chapitre de ce roman « sebba n tmacahut/l'argument du conte », ainsi que le motif traditionnel de l'homme ordinaire devenant saint par un concours extraordinaire des circonstances.

15. Cet ouvrage de N. Ait Ouali sur l'écriture romanesque en kabyle est le premier en son genre. Il est, à la fois, introductif et descriptif de quelques aspects du corpus romanesque kabyle. L'auteur y apporte son appréciation sur la qualité des ungal publiés (mais aussi sur des nouvelles) en s'intéressant à leur dimension romanesque à travers l'analyse de plusieurs niveaux tels la narration, les thématiques, le rapport à l'histoire, etc. Des études plus récentes proposent des visions d'ensemble de l'ungal en exposant, de manière générale, la question du roman amazigh (kabyle, rifain et chleuh). M. A. Salhi et $\mathrm{N}$. Sadi interrogent le roman berbère dans le champ littéraire maghrébin en abordant à la fois son corpus (d'un point de vue thématique) et son contexte d'apparition et d'évolution (conditions de production et de réception) (Salhi M. A. et Sadi N., 2016).

16. Il s'agit de Salas d Nuja de Brahim Tazaghart, de Bu-tqulhatin de Omar Dahmoune, de Ccna n yibzaz de Laifa Ait Bodaoud, etc.

17. "Nous pouvons envisager ce texte comme un roman car dans plusieurs de ses caractéristiques, il dépasse la nouvelle. Nous pouvons l'envisager comme une nouvelle car de facture courte, il repose sur une succession d'évènements ". C'est nous qui traduisons.

18. Il est possible de citer le cas des recueils de nouvelles Ameddakkel d tullisin niden et Tatabațața, respectivement de Mourad Zimu et de Mohand Arab Ait Kaci, qui arborent une généricité se rapprochant davantage du romanesque.

\section{RÉSUMÉS}

Cette étude se propose de suivre le discours critique ayant accompagné l'ungal/roman de langue kabyle et d'observer les jalons de son évolution au gré des enjeux du champ littéraire dans lequel 
il est produit. Il est question de voir la manière dont ces enjeux ont contribué à façonner les lectures qu'elles soient de l'ordre de la recherche universitaire ou s'inscrivant dans un cadre plus « informel » comme le cas des comptes rendus de lecture. L'objectif est de décrire la manière dont la réception s'est organisée pour répondre à certaines problématiques de l'espace social, à certains aspects contraignants du champ littéraire kabyle, et comment celle-ci s'est vue transformée une fois que ce même champ s'était doté de nouvelles données.

This study is devoted to the analyse of the critical discourse that accompanied L'ungal/the berber novel. It also observes the milestones of its evolution according to the challenges of the literary field in which it is produced. This study aims to find out how these issues have contributed to shape readings, whether they are of the order of the university research or part of a more "informal" setting such as the case of reading reports. The objective is to describe the way in which the reception was organized to respond to certain problems of the social space, to certain constraining aspects of the Kabyle literary field, and how it was transformed once the same field had new data.

\section{INDEX}

Mots-clés : Horizon d'attente, champ littéraire, positions, institutionnalisation, légitimation

Keywords : Horizon of expectation, literary field, positions, institutionalization, legitimation

\section{AUTEUR}

\section{NABILA SADI}

Département de langue et culture amazighes, Université M. Mammeri. Tizi Ouzou 